\section{Fundamentals of the fourth state}

C.N. Lashmore-Davies

Handbook of Plasma Physics, Vols I

and II.

Edited by A.A. Galeev and R.N. Sudan. North-Holland: 1983/1984. Vol. I

pp.751, \$180.75, Dfl.425. Vol. II pp.850, $\$ 190.50$, Dfl. 495.

AT A TIME when the growth in the publication of papers on plasma physics shows no sign of slowing, the appearance of two volumes which set out the basic ideas of the subject and provide summaries of all the main lines of research is particularly welcome. These two books are worthy successors to the Leontovich reviews and the Simon and Thompson series, and have the advantage of being published together. They contain 33 articles, 17 of which were contributed by 19 authors from the United States, 15 by 12 authors from the Soviet Union and one by two Japanese authors.

Volume I deals with the principles of plasma physics, and is divided into four parts. The first covers the motion of charged particles in electromagnetic fields, atomic collision processes relevant to plasmas and the various radiation phenomena which can occur in plasmas, concluding with an illuminating discussion of the different modes of description of plasmas for low frequency phenomena and an account of the subtleties of collisional transport in magnetically confined plasmas.

The remaining parts of Vol. I deal with the collective nature of plasmas. An important feature of a plasma is the great variety of waves it can support. The nature of the linearized wave motions allowed by ideal MHD and the effect of allowing for a distribution of particle velocities are both described. Also included are treatments of fluctuations, geometrical optics and the conversion of one linear wave into another in an inhomogeneous plasma.

When there is a reservoir of free energy in a plasma some of these wave modes can become unstable resulting in a great variety of instabilities. The many aspects of plasma instabilities from ideal and resistive MHD, the kinetic instabilities driven by the pressure gradient of a confined plasma, non-thermal particle distributions in velocity space and whether a given instability behaves like an amplifier or an oscillator are all systematically considered. An inevitable consequence of instability is that a plasma moves into a state in which its motion is non-linear. Since a plasma can support so many different wave motions it has been conjectured that a state of weak turbulence might exist, and in the final part of Vol. I the concepts behind this theory are lucidly set forth.

Volume II follows on from its companion, containing Parts 5-9, and is mainly

Off the record

concerned with non-linear consequences In many practical situations an enhanced level of long wavelength Langmuir waves can be generated (for example by an electron beam or a laser) which, according to weak turbulence theory, should relax to a coherent Langmuir oscillation. The resolution of this apparent paradox and the existence of three-dimensional, localized, high-frequency electric fields are beautifully described by the discoverer of the phenomenon. Also discussed are the spectra resulting from weak turbulence and the numerical modelling of strong Langmuir turbulence.

In hot plasmas the effect of binary collisions is often insufficient to explain various transport processes. The simplest of these phenomena is the dissipation of a current in an unmagnetized plasma, due to the generation of waves rather than interparticle collisions, although even this apparently simple example is not fully understood. Other examples considered are the dissipation of intense laser light and finite amplitude waves in plasmas through the non-linear generation of waves. Similar but far more complicated situations occur in the cross-field diffusion of particles and energy in magnetically confined plasmas. This complex issue is surveyed, together with an attempt at a comprehensive statistical description of plasma turbulence. These effects are generally referred to as anomalous although in many applications they represent the normal situation rather than the exception.

Part 8 consists of two excellent reviews on the diagnostic methods upon which the subject of plasma physics rests; the first is concerned with radiative techniques and the second with the methods used in hot magnetically confined plasmas, complementing those discussed in the first article. Volume II also contains an informative account of the methods of particle simulation by computer and a thorough discussion of the equilibrium and stability of nonneutral relativistic electron beams and their interaction with plasma.

Together, the books satisfy the aims of the general editors in encompassing most of the present-day knowledge of plasma physics, albeit from a user point of view. They are not suitable for someone requiring an introduction to the subject; although they take the reader to the frontiers of current research, they do not cover the general topic of the foundations of plasma kinetic theory nor do they contain more than a very brief account of the fundamental and fascinating subject of Landau damping. But those actively involved in research on plasma physics will find these volumes invaluable, as will experienced workers in other fields wishing to acquaint themselves with a particular aspect of the subject.

C.N. Lashmore-Davies is at the Culham Laboratory, Abingdon, Oxfordshire (Euratom/ UKAEA Fusion Association). C 1985 Nature Publishing Group

\section{John Parkington}

From Hunters to Farmers: The Causes and Consequences of Food Production in Africa.

Edited by $J$. Desmond Clark and Steven A. Brandt.

University of California Press: 1984.

Pp.433. \$63.25, £52.25.

AFRICA, as one of my mentors once sagely remarked, is a very big place. It encompasses environments as diverse as the sand seas of the Sahara and Namib, the thirstlands of the Kalahari and Sahel, the heath communities of the Cape and Mahgreb, the savannahs of Serengeti and the tropical forests of Zaire. To understand the social and ecological events leading to and following from the transition from food gathering to food production over such a vast and varied area is a Herculean task. Yet archaeologists are nothing if not optimists, and the goal of From Hunters to Farmers is nothing less than this. A collection of 30 papers, it sets out to provide a synthesis of current knowledge and is, in effect, the published proceedings of a symposium held in Los Angeles in 1978.

The volume is divided into four sections, the first consisting of three general papers which present a depressing but honest picture of how little we know about past climates, past population distributions and the circumstances surrounding the adoption of food production in Africa. Most of the contributions fall into the second section and are regional summaries of greater or lesser geographical scope. I wondered at this point what distinguishes papers that are quoted long after publication. Do they contain dazzling theoretical insights or essential primary observations? Or do they reflect the spirit of their times? Regional reconstructions based on modest but accumulating data sets are almost by definition transitory phenomena. We all write and then rewrite them.

To my mind the most interesting section is the third, in which the effects of the acquisition of domestic plants and animals by Kalahari San are documented. These three papers combine empirical observations with theoretical perspectives and will probably have longer lives than the regional accounts. Because they contribute to the realization that the San are, and have been, far from isolated from agriculturalists and pastoralists, they will have immediate im-

\section{New in paperback}

- The Creative Computer: Machine Intelligence and Human Knowledge by Donald Michie and Rory Johnston. Publisher is Penguin, price is 66.95. For review see Nature 313, 77 (1985).

- When the Snakes Awake: Animals and Earthquake Prediction by Helmut Tributsch. Publisher is MIT Press, price is $\$ 8.85, £ 9.25$. For review see Nature 302, 763 (1983). 